\title{
Open Repair of the Triangular Fibrocartilage Complex from Palmar Aspect
}

\author{
Hisao Moritomo, MD, $\mathrm{PhD}^{1}$ \\ 1 Yukioka Hospital Hand Center, Osaka Yukioka College of Health \\ Science, Osaka, Japan \\ J Wrist Surg 2015;4:2-8. \\ Address for correspondence Hisao Moritomo, MD, PhD, Yukioka \\ Hospital Hand Center, Osaka Yukioka college of Health Science, 2-2-3, \\ Ukita, Kita-ku, Osaka-shi, Osaka 530-0021, Japan \\ (e-mail: hisao-moritomo@yukioka-u.ac.jp).
}

\begin{abstract}
Keywords

- DRUJ instability

- TFCC foveal tear

- open repair

- CT arthrography

- surgical approach

Background Although foveal tears of the triangular fibrocartilage complex (TFCC) can be surgically reattached to the fovea via a dorsal approach, the foveal lesion is difficult to visualize from the dorsal side because the extensor carpi ulnaris (ECU) sheath floor and the superficial dorsal limb of the radioulnar ligament hinders the view of the fovea. Materials and Methods Twenty-one patients with foveal tears were treated by an open repair method from the palmar aspect. Pain, instability, motion, and grip strength were evaluated after a mean follow-up period of 26 months, and each patient was rated according to the Mayo Modified Wrist Score (MMWS).

Description of Technique A 4-cm skin incision is made on the palmar aspect of the ulnar fovea. The ulnar fovea is exposed through a transverse capsulotomy of the distal radioulnar joint. The distal aspect of the TFCC is also exposed between the ECU tendon sheath and the ulnotriquetral ligament. After curettage of the scar tissue in the fovea, the deep palmar and dorsal limbs of the TFCC are sutured back to the fovea using a suture anchor technique.

Results Foveal TFCC tears could be repaired via a palmar surgical approach without violating the floor of the ECU tendon sheath and the superficial dorsal limb. Excellent results were achieved in 18 patients, and a good result was achieved in three.

Conclusions Our result compared favorably with those reported for dorsal approach. The palmar surgical approach facilitates the inspection and repair of the TFCC foveal tears.

Level of Evidence IV
\end{abstract}

Instability of the distal radioulnar joint (DRUJ) caused by a traumatic foveal tear of the triangular fibrocartilage complex (TFCC) can produce significant disability, which could present as pain, weakness, and a restricted range of motion. Several surgical procedures to repair TFCC foveal tear have been proposed, including open ${ }^{1-4}$ and arthroscopic $^{5-9}$ TFCC repairs. Several surgical approaches have also been proposed, including a dorsal ${ }^{10-12}$ or a palmar ${ }^{3,10,13,14}$ approach. However, the ideal procedure and approach for TFCC foveal tear remain controversial.

Over the past few decades, a considerable number of studies of the anatomy of the TFCC have been undertaken. ${ }^{15-19}$ As each palmar and dorsal radioulnar ligament extends toward the ulna, it divides into two limbs: the deep limb attaches to the fovea on the ulna, and the superficial limb attaches to the base and midportion of the ulnar styloid. ${ }^{15-19}$ Thus, the TFCC has four insertions on the ulna, which can be termed the deep palmar limb, deep dorsal limb, superficial palmar limb, and superficial dorsal limb ${ }^{2}$ (-Fig. 1).

Regarding the direction of DRUJ instability, palmar radius instability, in which the distal radius is unstable in a palmar direction with respect to the ulna, is a much more common form of DRUJ instability than dorsal radius instability. ${ }^{20} \mathrm{~A}$ biomechanical study showed that the deep palmar limb has a
Copyright (c) 2015 by Thieme Medical Publishers, Inc., 333 Seventh Avenue, New York, NY 10001, USA. Tel: +1(212) 584-4662
DOI http://dx.doi.org/ 10.1055/s-0034-1398474. ISSN 2163-3916. 


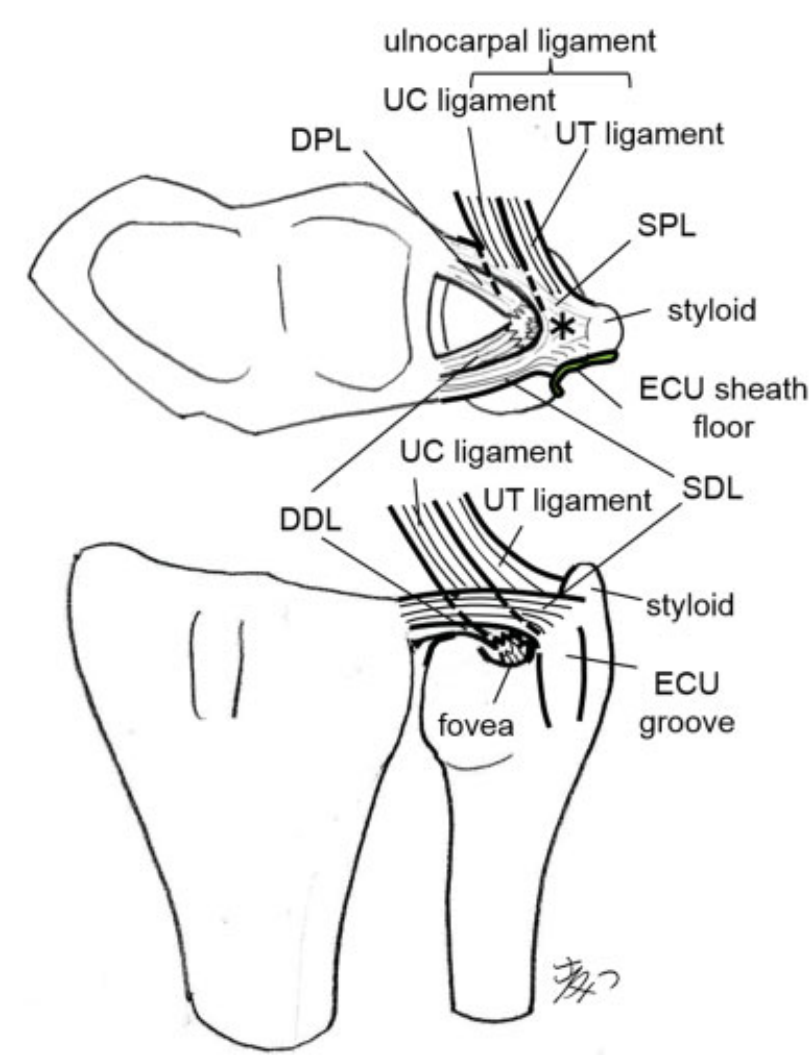

Fig. 1 Schematic representation of foveal avulsion of TFCC, in which its foveal insertion is disrupted, leaving some styloid insertion intact. DDL, deep dorsal limb; DPL, deep palmar limb; ECU, extensor carpi ulnaris; SDL, superficial dorsal limb; SPL, superficial palmar limb; UC, ulnocapitate; UT, ulnotriquetrum; *, common juncture of the palmar and dorsal radioulnar ligament. The articular disk and ulnolunate ligament are not drawn.

greater constraint percentage to palmar radius instability than the deep dorsal limb does. ${ }^{21}$ Therefore, I considered that repairing the foveal insertion of the deep palmar limb was critical to managing a TFCC foveal tear in cases with palmar radius instability (-Fig. $\mathbf{2 a}$, b).

TFCC foveal tears have been surgically reattached to the fovea via a dorsal approach with generally good clinical results. ${ }^{1,2,4}$ In the authors' experience, when the TFCC was repaired through a dorsal approach, I often found that the foveal lesion was difficult to visualize from the dorsal side because the superficial dorsal limb of the radioulnar ligament and the extensor carpi ulnaris (ECU) sheath floor hindered the view of the fovea ${ }^{14}$ (-Fig. 3a). It was necessary to incise the superficial dorsal limb and the ECU sheath floor to inspect the foveal lesion, which increased the DRUJ instability. An alternative palmar surgical approach ${ }^{14}$ was therefore developed to obtain satisfactory views of the disrupted foveal insertions and to avoid iatrogenic injury to the superficial dorsal limb and the ECU sheath floor.

- The purpose of this study is to describe surgical technique of open repair of the TFCC from the palmar aspect and to assess the operative findings and the clinical results of the palmar open repair method.

\section{Patients and Methods}

Between 2003 and 2014, 37 patients with traumatic foveal TFCC tears were surgically treated by a single surgeon (HM) using an open method via either a palmar or a dorsal approach. Patients with a fresh or chronic TFCC foveal tear with a dynamic DRUJ instability were candidates for the open repair procedure. Eight patients who were repaired via a dorsal approach were excluded from this study. I used dorsal approach not only for dorsal radius instability but for palmar radius instability with predominant dorsal pain, which was thought to be indicative of a superficial dorsal limb avulsion, ECU tendinitis, or both in addition to foveal avulsion. The palmar approach was chosen for 29 patients, essentially with predominant palmar tenderness, which was thought to be indicative of a predominantly palmar foveal avulsion rather than a dorsal avulsion. Eight patients with a static DRUJ instability were treated by a palmar TFCC reconstruction method ${ }^{20}$ and were excluded in this study. The remaining 21 patients who were treated with a palmar TFCC repair were included in this study. A total of 13 men and 8 women with a mean age of 31 years (range, 14-52 years) were evaluated. All 21 patients presented with ulnar sided wrist pain. The injury mechanism was forced wrist extension in 14 patients and forced forearm supination in 7 patients. None of the patients had a
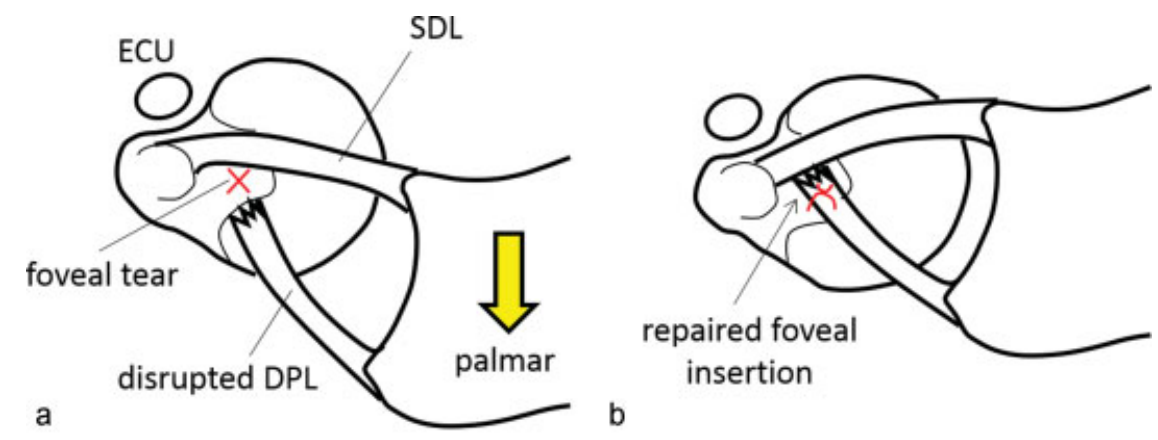

Fig. 2a, b (a) The palmar radius instability, in which the distal radius is unstable to the palmar direction with respect to the ulna, is a much more common DRUJ instability than the dorsal radius instability is. The DPL is much more resistant against the palmar radius instability than the deep dorsal limb. (b) Repairing the foveal insertion of the deep palmar limb is critical to manage TFCC foveal tear. The deep dorsal limb and the superficial palmar limb are not drawn. 


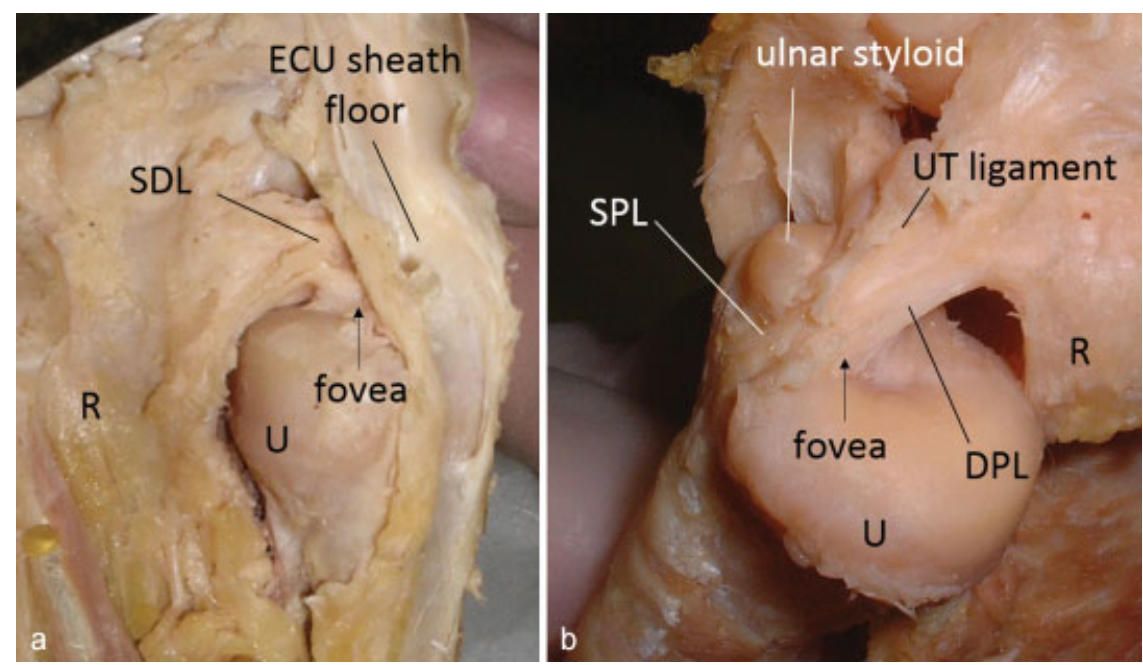

Fig. 3a, b Ulnar insertions of the TFCC on a cadaver specimen (right wrist). (a) TFCC seen from the dorsal side of the ulna. The SDL and the floor of the extensor carpi ulnaris (ECU) tendon sheath hinder the view of the fovea. (b) The foveal insertion seen from the palmar side of the ulna. Note that TFCC insertions to the fovea and the ulnar styloid are clearly visualized. R: radius, U: ulna.

history of a distal radius fracture. A positive fovea sign, ${ }^{22}$ which is tenderness in the ulnar snuff box between the extensor and flexor carpi ulnaris (FCU) tendons, was found in 16 of the 21 patients. I used the DRUJ ballottement test in neutral forearm position to evaluate DRUJ instability. All patients had palmar radius instability, and no patients had dorsal radius instability. Mild, moderate, and severe palmar radius instability were found in 5, 13, and 3 patients, respectively. Presence of ulnar impaction or ulnocarpal impingement was tested by the shake hand test. ${ }^{23}$ In the shake hand test, the patient is asked to grip the examiner's hand with the patient's elbow in $90^{\circ}$ of flexion and the forearm in neutral rotation. The shake hand test is positive when pain occurs during passive ulnar deviation of the patient's wrist by the examiner. Positive shake hand test was found in 13 patients. Presence of lunotriqueral ligament tears was also tested by lunotriqueral ballottement shear test. Positive lunotriqueral ballottement shear test was found in 3 patients.

The diagnosis was initially made using magnetic resonance imaging (MRI) or arthrograms of the DRUJ. MRI revealed a high-intensity area on T2-weighted images at the fovea. and the arthrograms showed pooling of the dye at the fovea. Since 2008, we have been using computed tomography (CT) arthrography to detect foveal tears. In addition, we developed a new CT view called radial plane view, in which the image slices rotate clockwise with the center of rotation on the ulnar styloid process ( - Fig. 4a). Using the radial plane views, we obtained more than 10 slices through the foveal lesion, whereas only 4-5 slices were obtained in the coronal view. Moreover, with the radial plane views, we obtained images of the total length of the palmar and dorsal radioulnar ligaments, which attach to the fovea and the ulnar styloid ( $\mathbf{- F i g s . ~} \mathbf{4 b}, \mathbf{c}$ ). The radial plane views were helpful for the diagnosis of foveal tears, especially for detecting whether tears occurred predominantly at the palmar or dorsal side, compared with the conventional coronal plane views alone. Lunotriqueral membranous tears were found on MRI or CT arthrography in three cases.

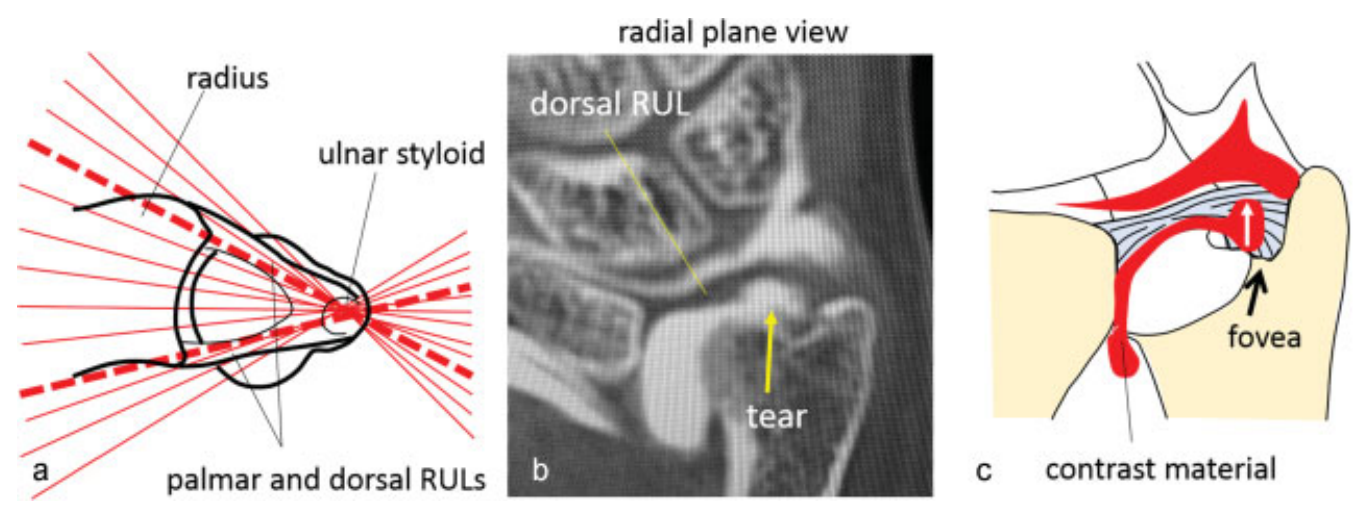

Fig. 4a-c (a) A schema illustrating the relationship between the slice planes of the radial plane view and the radioulnar ligaments (RULs). The image slices rotate clockwise with the center of rotation on the ulnar styloid process. Note that some slices were collected along the course of the palmar and dorsal RULs (thick dotted lines). (b) Radial plane view of CT arthrography of a case with a foveal tear. A roundish defect in the ulnar portion of the deep fiber of the RUL that inserts at the fovea, leaving the styloid insertion of TFCC intact. The contrast material injected into DRUJ delineated a disrupted site at the immediate radial aspect of the fovea. (c) A schema illustrating common type of foveal tear. 
The preoperative arc of wrist extension/flexion averaged $135^{\circ} \pm 32^{\circ}$, and that of pronation/supination averaged $142^{\circ} \pm 22^{\circ}$. Preoperative grip strength averaged $25 \pm 11 \mathrm{~kg}$, which was $65 \% \pm 20 \%$ of the unaffected side. Duration of symptoms before surgery averaged $11 \pm 15$ months. The study was approved by the local ethics committee, and informed consent was obtained from all patients.

\section{Surgical Technique}

This procedure was performed with the patient under general anesthesia or a regional block and in a supine position with the shoulder abducted at $90^{\circ}$. The elbow was flexed at $90^{\circ}$ with the forearm in a neutral position or extended with the forearm supinated on the arm board. A 4-cm curved or zigzag skin incision was made along the FCU tendon on the medial aspect of the pisiform (-Fig. 5a). The FCU tendon and the main trunk of the ulnar nerve were retracted radially (-Fig. 5b), while the dorsal cutaneous ulnar branch was retracted ulnarly without being separated from the skin. The deep fascia that connects the ECU tendon sheath with the pisiform was incised. A transverse incision was made in the palmar capsule of the DRUJ from the ulnodistal corner of the palmar sigmoid notch of the radius to the base of the ulnar styloid at the distal margin of the ulnar head (-Fig. 5c). The foveal lesion can be clearly visualized by retracting the disrupted TFCC distally. The medial margin of the ulnocarpal ligamentous complex (UCLC) ${ }^{17}$ is easy to identify by palpation and was exposed by excising the soft spot of the medial ulnocarpal joint capsule, which is located between the ulnotriquetral ligament and ECU sheath floor. ${ }^{24}$ Excision of the pisotriquetral joint capsule also helps to identify the UCLC. The foveal and styloid insertions were carefully inspected from both the proximal and distal sides of the TFCC (-Figs. 6a, b). We especially focused on the condition of fibers of the four radioulnar ligaments: the deep palmar limb, the deep dorsal limb, the superficial palmar limb, and the superficial dorsal limb. We judged each limb to be disrupted when its ulnar insertion was discontinued or replaced by loose scar tissue. The superficial dorsal limb was usually found intact and left as is. After the scar tissue is curetted, the avulsed common juncture of the palmar and dorsal radioulnar ligament and the articular disc were sutured down onto the fovea using a suture anchor (2.7-mm Ti Screw Suture Anchor, Biomet Sports Medicine, Inc., Warsaw, IN, USA, or 2.8-mm Twinfix, Smith \& Nephew Orthopaedics KK, Tokyo, Japan) (-Fig. 6c-f, 7). An ulnar shortening operation was simultaneously performed in 11 patients who had a $+3-\mathrm{mm}$ ulnar variance or severe pain by the shake hand test. In the ulnar shortening operation, two parallel transverse osteotomies were performed $\sim 6 \mathrm{~cm}$ proximal from the distal end of the ulna, and 2 to $3 \mathrm{~mm}$ of bone are removed. A six-hole, 2.7- or 3.5-mm, dynamic compression plate was held over the distal third of the ulna. A long arm cast or a sugar tong splint was applied postoperatively with the forearm in the neutral position for 3 weeks, after which the cast was replaced by a removable wrist splint for 8 weeks. Strengthening and resumption of activities were delayed until nearpainless normal range of motion was reported.

\section{Clinical Findings}

Pain, instability, motion (flexion/extension and pronation/ supination), and grip strength were evaluated after a mean follow-up period of 26 months (range, 6-65 months), and each patient was rated according to the Mayo Modified Wrist Score (MMWS) System. ${ }^{25}$ The degree of pain was divided into four classes: none, mild, moderate (tolerable), and severe (intolerable). ${ }^{25}$ Postoperative pain was also evaluated by visual analog scale (VAS) between 0 and 10. The degree of instability was divided into four classes ${ }^{3}$ : none (same as contralateral side), mild (more unstable than contralateral side but not manually subluxed), moderate (subluxed but not manually dislocated), and severe (manually dislocated). The Wilcoxon rank sum test was used to compare the differences from the MMWSs.
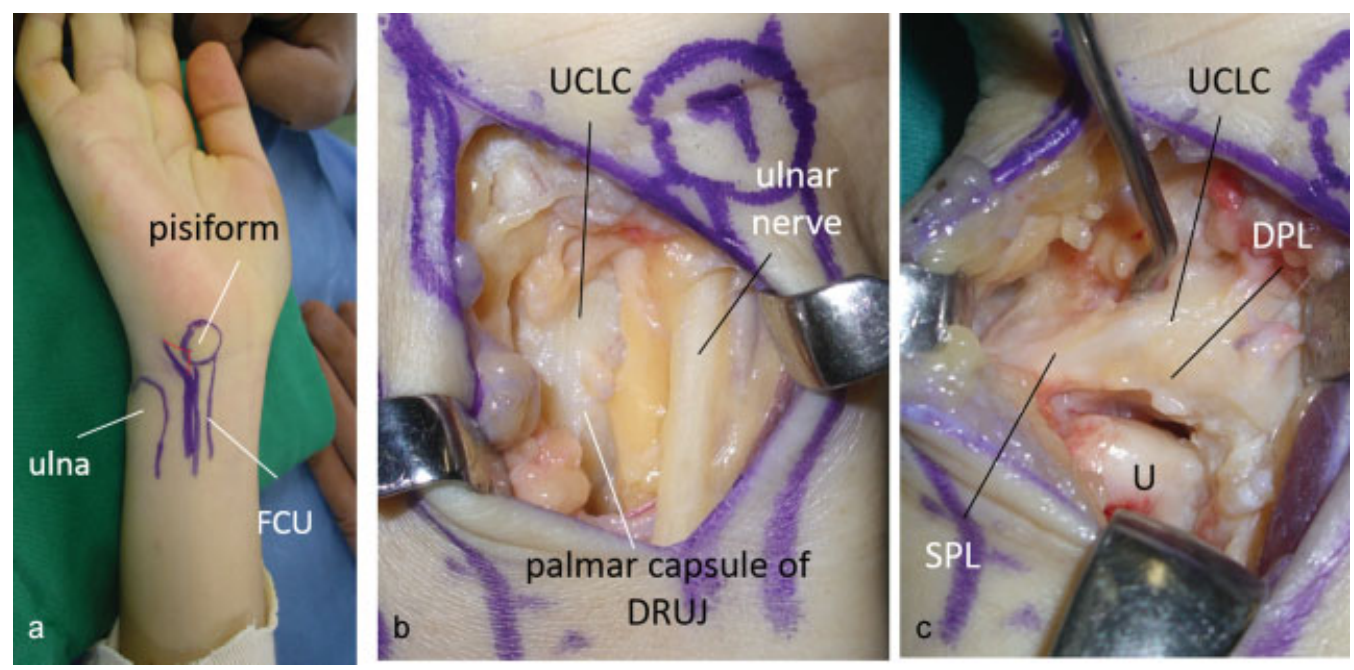

Fig. 5a-c (a) Skin incision for a palmar approach (right wrist). (b) The ulnar nerve and the dorsal cutaneous branch of the ulnar nerve are retracted radially and ulnarly, respectively. (c) Palmar view of the TFCC. The palmar deep limb (PDL) is disrupted, but the palmar superficial limb (PSL) is intact. 

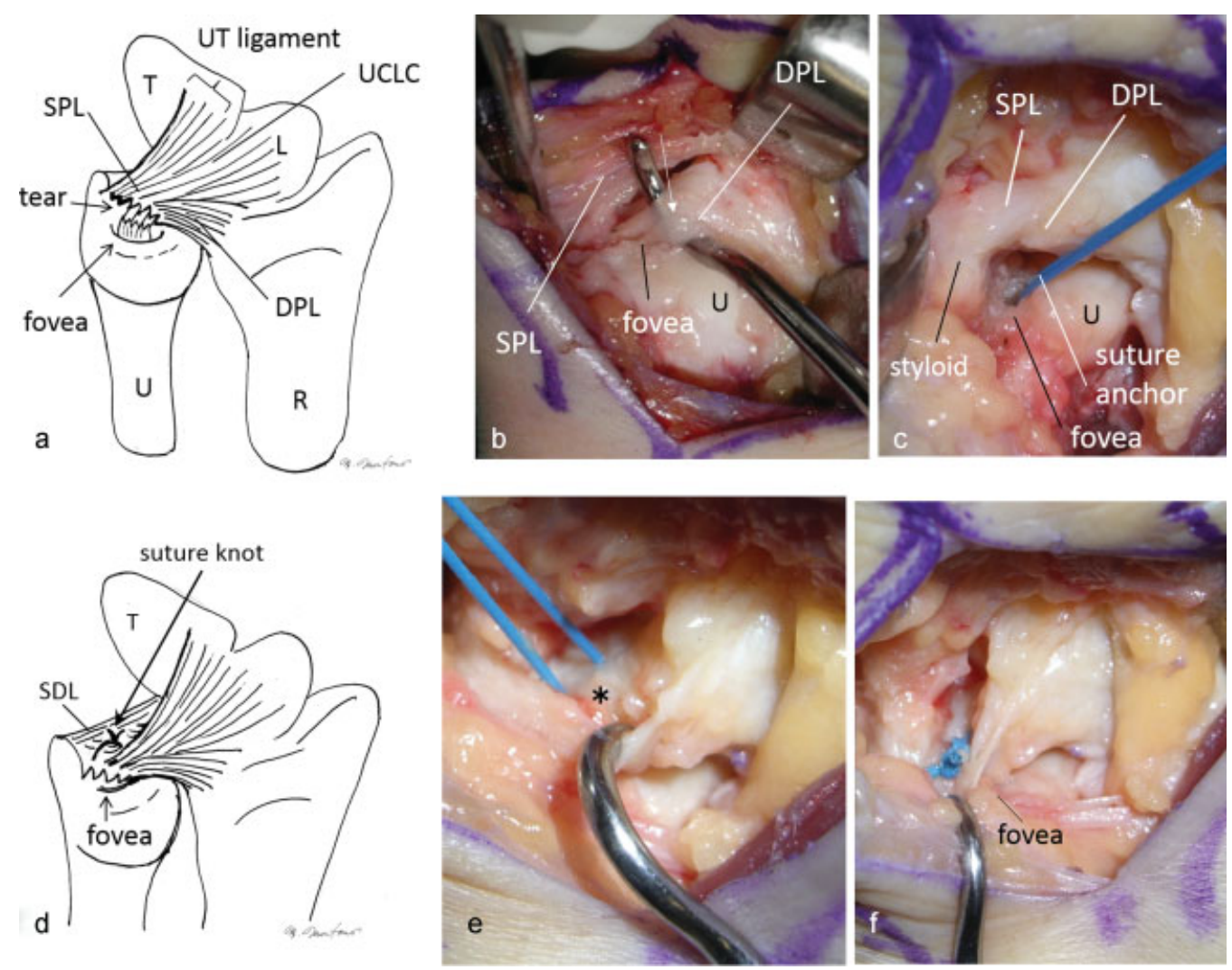

Fig. 6a-f Palmar view of the most common TFCC foveal tear via a palmar approach (right wrist). (a, b) The DPL is disrupted and replaced by loose scar tissue (arrow). (c) After curettage of the scar, a suture anchor is inserted at the fovea. (d) Two 2-0 nonabsorbable threads from the anchor are passed through the common juncture of the palmar and dorsal radioulnar ligament $\left(^{*}\right)$ from proximal to distal. (e, f) A suture knot is tied distally, and the lifted TFCC is sutured tightly down onto the fovea. The SDL is left as it is.

\section{Results}

The foveal insertions of the TFCC were found to be disrupted and replaced by loose scar tissue in all 21 patients. The deep palmar limb was disrupted in all patients. The deep dorsal limb was disrupted in 19 patients. The superficial dorsal limb was not disrupted in any patient. The superficial palmar limb was disrupted in 15 patients.

Eleven patients denied any pain whatever, nine patients complained only of mild pain, and one patient complained of moderate pain. Mean postoperative VAS score was 2.0 (range $0-5)$. The final clinical results of motion and grip strength showed postoperative significant improvement (-Table $\mathbf{1}$ ). The postoperative arc of wrist extension/flexion averaged $160^{\circ} \pm 14^{\circ}(p<0.01)$, and that of pronation/supination averaged $156^{\circ} \pm 14^{\circ} \quad(p<0.001)$. Postoperative grip strength averaged $35 \mathrm{~kg} \pm 11 \mathrm{~kg}(p<0.001)$, which was $92 \% \pm 10 \%$ of the unaffected side. The MMWSs significantly improved postoperatively from $43 \pm 16$ to $92 \pm 6$ $(p<0.001)$. Excellent results were achieved in 18 patients, and a good result was achieved in three. No, mild, moderate, and severe DRUJ instability were found in $16,4,1$, and 0 patients, respectively. In this series, the times to surgery of the three patients with good results were 2, 3, and 9 months, and the time to surgery did not influence the outcome. Irritation of the cutaneous branch of the ulnar nerve occurred in 10 patients postoperatively but resolved within a few weeks. There was one delayed union and no nonunion after ulnar shortening.

\section{Discussion}

The deep palmar limb is biomechanically much more important in preventing palmar radius instability than the deep dorsal limb is. ${ }^{21}$ By contrast, the deep dorsal limb is not so important in preventing dorsal radius instability because the distal interosseous membrane prevents dorsal radius instability if the deep dorsal limb is cut. ${ }^{21}$ The superficial dorsal limb is also important because several biomechanical studies have suggested that the superficial dorsal limb and the deep palmar limb become taut in pronation. ${ }^{26,27}$ Therefore, I considered that repairing the foveal insertion of the deep palmar limb and avoiding iatrogenic violation of the superficial dorsal limb were critical to managing a TFCC foveal tear in cases with palmar radius instability. We found the palmar surgical approach facilitates the inspection and repair of the TFCC foveal tears. Our result compared favorably with those reported for the dorsal approach.

The limitations of this study were the small sample size, noncomparative study, and the retrospective nature. Eleven patients underwent simultaneous ulnar shortening. It is therefore difficult to know whether the foveal attachment 
Table 1 Pre- and postoperative clinical data

\begin{tabular}{|l|l|l|}
\hline & Preoperative & Postoperative \\
\hline Arc of extension/flexion & $135^{\circ} \pm 32^{\circ}$ & $160^{\circ} \pm 14^{\circ} \dagger$ \\
\hline Arc of pronation/supination & $142^{\circ} \pm 22^{\circ}$ & $156^{\circ} \pm 14^{\circ}$ \\
\hline Grip strength (\% of unaffected side) & $25 \pm 11 \mathrm{~kg}(65 \% \pm 20 \%)$ & $35 \mathrm{~kg} \pm 11 \mathrm{~kg} *(92 \% \pm 10 \%)$ \\
\hline Mayo Modified Wrist Score & $43 \pm 16$ & $92 \pm 6^{*}$ \\
\hline
\end{tabular}

${ }^{\dagger} p<0.01,{ }^{*} p<0.001$

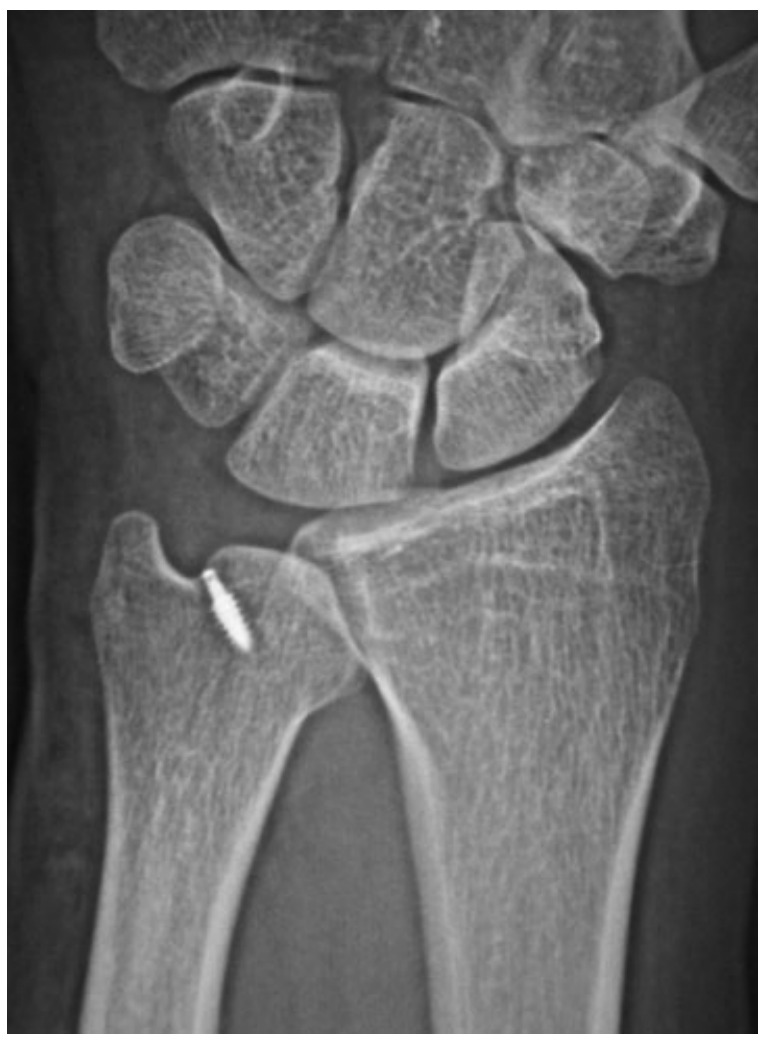

Fig. 7 Postoperative X-ray image showing a suture anchor inserted into the fovea.

of TFCC via a palmar approach or the ulnar shortening was responsible for the resolution of the patients' symptoms.

A wide variation in the magnitude of DRUJ instability due to TFCC foveal tear has been observed, ranging from dynamic instability, in which the radius does not dislocate in the resting position but dislocates when the examiner passively translates the radius palmarly or dorsally with respect to the ulna, to static instability, in which the radius is extremely unstable and dislocates with respect to the ulna in the resting position. Static DRUJ instability cannot be restored by simple suture of the TFCC and often requires reconstruction of the palmar and dorsal radioulnar ligaments. ${ }^{20,28-30}$

TFCC foveal tears have been surgically reattached to the fovea by open techniques via a dorsal approach with generally good clinical results. ${ }^{1,2,4}$ Hermansdorfer et al ${ }^{1}$ reported that among 11 patients with surgical reattachment for TFCC avulsion, eight patients returned to essentially normal painless activity, but three patients had unsatisfactory results, two of whom needed subsequent surgery such as a distal ulna resection. Chou et $\mathrm{al}^{2}$ reported that 3 of $8(37.5 \%)$ patients had an excellent result and $5(62.5 \%)$ had a good result according to the MMWS. Nakamura et $\mathrm{al}^{4}$ reported $56(84.8 \%)$ excellent, 6 (9.1\%) good, $2(3.0 \%)$ fair, and $2(3.0 \%)$ poor results among 66 patients according to their original DRUJ-evaluating system. In this current study, excellent results were achieved in 18 of 21 (85.7\%), a good result was achieved in 3 (14.3\%). Our result compared favorably with those reported for a dorsal approach.

Thus, we considered that the palmar approach is beneficial for repairing TFCC foveal tear to obtain satisfactory views of the disrupted foveal insertion of both the deep palmar and the dorsal limb and to avoid iatrogenic violation of the superficial dorsal limb and the ECU sheath floor.

\section{Conflict of Interest \\ None}

\section{References}

1 Hermansdorfer JD, Kleinman WB. Management of chronic peripheral tears of the triangular fibrocartilage complex. J Hand Surg Am 1991;16(2):340-346

2 Chou KH, Sarris IK, Sotereanos DG. Suture anchor repair of ulnarsided triangular fibrocartilage complex tears. J Hand Surg $[\mathrm{Br}]$ 2003;28(6):546-550

3 Moritomo H, Masatomi T, Murase T, Miyake J, Okada K, Yoshikawa $\mathrm{H}$. Open repair of foveal avulsion of the triangular fibrocartilage complex and comparison by types of injury mechanism. J Hand Surg Am 2010;35(12):1955-1963

4 Nakamura T, Sato K, Okazaki M, Toyama Y, Ikegami H. Repair of foveal detachment of the triangular fibrocartilage complex: open and arthroscopic transosseous techniques. Hand Clin 2011;27(3): 281-290

5 Luchetti R, Atzei A, Cozzolino R, Fairplay T, Badur N. Comparison between open and arthroscopic-assisted foveal triangular fibrocartilage complex repair for post-traumatic distal radio-ulnar joint instability. J Hand Surg Eur Vol 2014;39(8): 845-855

6 Yao J, Lee AT. All-arthroscopic repair of Palmer 1B triangular fibrocartilage complex tears using the FasT-Fix device. J Hand Surg Am 2011;36(5):836-842

7 Geissler WB. Arthroscopic knotless peripheral ulnar-sided TFCC repair. Hand Clin 2011;27(3):273-279

8 Iwasaki N, Nishida K, Motomiya M, Funakoshi T, Minami A. Arthroscopic-assisted repair of avulsed triangular fibrocartilage 
complex to the fovea of the ulnar head: a 2- to 4-year follow-up study. Arthroscopy 2011;27(10):1371-1378

9 Shinohara T, Tatebe M, Okui N, Yamamoto M, Kurimoto S, Hirata H. Arthroscopically assisted repair of triangular fibrocartilage complex foveal tears. J Hand Surg Am 2013;38(2):271-277

10 Garcia-Elias M, Hagert E. Surgical approaches to the distal radioulnar joint. Hand Clin 2010;26(4):477-483

11 Garcia-Elias M, Smith DE, Llusá M. Surgical approach to the triangular fibrocartilage complex. Tech Hand Up Extrem Surg 2003;7(4):134-140

12 Nakamura T, Nakao Y, Ikegami H, Sato K, Takayama S. Open repair of the ulnar disruption of the triangular fibrocartilage complex with double three-dimensional mattress suturing technique. Tech Hand Up Extrem Surg 2004;8(2):116-123

13 Kleinman WB, Graham TJ. The distal radioulnar joint capsule: clinical anatomy and role in posttraumatic limitation of forearm rotation. J Hand Surg Am 1998;23(4):588-599

14 Moritomo H. Advantages of open repair of a foveal tear of the triangular fibrocartilage complex via a palmar surgical approach. Tech Hand Up Extrem Surg 2009;13(4):176-181

15 Berger RA. The ligaments of the wrist. A current overview of anatomy with considerations of their potential functions. Hand Clin 1997;13(1):63-82

16 Ishii S, Palmer AK, Werner FW, Short WH, Fortino MD. An anatomic study of the ligamentous structure of the triangular fibrocartilage complex. J Hand Surg Am 1998;23(6):977-985

17 Garcia-Elias M. Soft-tissue anatomy and relationships about the distal ulna. Hand Clin 1998;14(2):165-176

18 Haugstvedt JR, Berger RA, Nakamura T, Neale P, Berglund L, An KN. Relative contributions of the ulnar attachments of the triangular fibrocartilage complex to the dynamic stability of the distal radioulnar joint. J Hand Surg Am 2006;31(3):445-451
19 Kleinman WB. Stability of the distal radioulna joint: biomechanics, pathophysiology, physical diagnosis, and restoration of function what we have learned in 25 years. J Hand Surg Am 2007;32(7): 1086-1106

20 Moritomo H, Kataoka T. Palmar reconstruction of the triangular fibrocartilage complex for static instability of the distal radioulnar joint. Tech Hand Up Extrem Surg 2014;18(3):110-115

21 Stuart PR, Berger RA, Linscheid RL, An KN. The dorsopalmar stability of the distal radioulnar joint. J Hand Surg Am 2000; 25(4):689-699

22 Tay SC, Tomita K, Berger RA. The "ulnar fovea sign" for defining ulnar wrist pain: an analysis of sensitivity and specificity. J Hand Surg Am 2007;32(4):438-444

23 Moritomo H. Differential diagnosis of ulnar wrist pain. Orthopaedics 2014;27:1-7 (Japanese)

24 Moritomo H. Anatomy and clinical relevance of the ulnocarpal ligament. J Wrist Surg 2013;2(2):186-189

25 Cooney WP, Linscheid RL, Dobyns JH. Triangular fibrocartilage tears. J Hand Surg Am 1994;19(1):143-154

26 Hagert CG. Distal radius fracture and the distal radioulnar jointanatomical considerations. Handchir Mikrochir Plast Chir 1994; 26(1):22-26

$27 \mathrm{Xu} \mathrm{J}$, Tang JB. In vivo changes in lengths of the ligaments stabilizing the distal radioulnar joint. J Hand Surg Am 2009;34(1):40-45

28 Adams BD, Berger RA. An anatomic reconstruction of the distal radioulnar ligaments for posttraumatic distal radioulnar joint instability. J Hand Surg Am 2002;27(2):243-251

29 Scheker LR, Ozer K. Ligamentous stabilization of the distal radioulnar joint. Tech Hand Up Extrem Surg 2004;8(4):239-246

30 Teoh LC, Yam AK. Anatomic reconstruction of the distal radioulnar ligaments: long-term results. J Hand Surg [Br] 2005;30(2): 185-193 